\title{
Presencia de Pseudosuccinea columella naturalmente infestada con Fasciola hepatica en Santo Tomé (Corrientes, Argentina)
}

\author{
Moriena, R.A. ${ }^{\text {; }}$ Alvarez, J.D. ${ }^{1}$; Pietrokovsky, S. ${ }^{2}$; Rubel, D. ${ }^{2}$; Prepelitchi, L. ${ }^{2}$; \\ Racioppi, O. ${ }^{1}$; Wisnivesky, C. ${ }^{2}$ \\ ${ }^{1}$ Cátedra Enfermedades Parasitarias, Facultad de Ciencias Veterinarias, UNNE, Sargento Cabral 2139, \\ Corrientes (3400), Argentina, Tel/fax 03783-425753. ${ }^{2}$ Departamento Ecología, Genética y Evolución, Facultad \\ de Ciencias Exactas y Naturales, UBA. E-mail: rmoriena@vet.unne.edu.ar
}

\begin{abstract}
Resumen
Moriena, R.A.; Alvarez, J.D.; Pietrokovsky, S.; Rubel, D.; Prepelitchi, L.; Racioppi, O.; Wisnivesky, C.: Presencia de Pseudosuccinea columella naturalmente infestada con Fasciola hepatica en Santo Tomé (Corrientes, Argentina). Rev. vet. 19: 2, 147-149, 2008. La fasciolosis es una enfermedad producida por Fasciola hepatica. Esta parasitosis produce grandes pérdidas económicas en el ganado bovino de la Provincia de Corrientes, Argentina, y también tiene carácter zoonótico. En el norte de la Provincia, Pseudosuccinea columella es el caracol involucrado en la transmisión de la enfermedad. El objetivo del presente trabajo fue ampliar los datos sobre la distribución de $P$. columella y su infestación natural por $F$. hepatica en la Provincia de Corrientes. Se recolectaron caracoles en un establecimiento ganadero del Departamento de Santo Tomé, al nordeste de la Provincia, que fueron identificados taxonómicamente, medidos y disecados para verificar la presencia de cercarias de $F$. hepatica. Los 115 caracoles recolectados fueron identificados como $P$. columella. La longitud de la conchilla varió entre $0,8-21,3 \mathrm{~mm}$, con una media de $10,0 \mathrm{~mm}$. La prevalencia de caracoles naturalmente infestados por $F$. hepatica fue de $8(7,4 \%)$ sobre 108 examinados. La presencia de $P$. columella en distintos departamentos del norte y nordeste de la Provincia de Corrientes y las altas prevalencias de infestación natural por $F$. hepatica registradas, indicarían el asentamiento de un ciclo de transmisión enzoótica en esta zona.
\end{abstract}

Palabras clave: Pseudosuccinea columella, Fasciola hepatica, infestación, prevalencia.

\begin{abstract}
Moriena, R.A.; Alvarez, J.D.; Pietrokovsky, S.; Rubel, D.; Prepelitchi, L.; Racioppi, O.; Wisnivesky, C.: Presence of Pseudosuccinea columella naturally infested by Fasciola hepatica in Santo Tomé (Corrientes, Argentina). Rev. vet. 19: 2, 147-149, 2008. Fasciolosis is a parasitic disease caused by Fasciola hepatica, that originates great economic losses in livestock production and is also considered a zoonosis. In Corrientes, northeastern Argentine, the snail Pseudosuccinea columella is involved in the transmission of the disease. The objective of this work is to provide with up-do-date data on the distribution and presence of naturally infested P. columella snails in this region. Snails were collected from a cattle farm in Santo Tomé, northeast of Corrientes. Snails were taxonomically classified, measured and examined for $F$. hepatica cercariaes. All 115 snails were identified as $P$. columella. Shell length ranged between $0.8-21.3 \mathrm{~mm}$, with a mean of $10.0 \mathrm{~mm}$. Prevalence of $F$. hepatica infestation was 8 (7.4\%) over 108 snails. The presence of $P$. columella and the high prevalence values recorded for the northern and northeastern areas of Corrientes may indicate the establishment of an enzootic transmission cycle in the region.
\end{abstract}

Key words: Pseudosuccinea columella, Fasciola hepatica, infestation, prevalence.

\section{INTRODUCCIÓN}

La fasciolosis hepática es una zoonosis producida por Fasciola hepatica (Trematoda: Digenea). La enfermedad produce grandes pérdidas económicas en el ganado bovino de la Provincia de Corrientes. Uno de los huéspedes intermediarios de este trematodo es Pseudo-

Recibido: 22 setiembre 2008 / Aceptado: 17 noviembre 2008 succinea columella, un caracol pulmonado de la familia Lymnaeidae (Figura 1).

En Sudamérica $P$. columella (=Lymanea columella) fue hallada en Ecuador ${ }^{15}$, Brasil ${ }^{15,24}$, Paraguay ${ }^{8}$, Uruguay ${ }^{5}$, Colombia ${ }^{20}$, Venezuela ${ }^{11}$, Perú ${ }^{9}$ y Argentina 3,22. Las publicaciones que mencionan caracoles de esta especie naturalmente infestados con $F$. hepatica son escasas, habiéndose reportado en Brasil 4, 6, 19, 21, 23, 25 , Argentina $^{18}$, Uruguay ${ }^{7}$, Perú ${ }^{9}$ y Colombia ${ }^{20}$. 
En razón a los antecedentes mencionados, el objetivo principal del presente trabajo fue aportar datos sobre la distribución de $P$. columella y su importancia como huésped intermediario de $F$. hepatica en la Provincia de Corrientes, Argentina.

\section{MATERIAL Y MÉTODOS}

En Septiembre de 2003 se recolectaron caracoles en un pequeño canal artificial de 1.000 metros de largo aproximadamente, que desaguaba en el Río Aguapey, dentro de un establecimiento ganadero situado en el Departamento Santo Tomé, al nordeste de la Provincia de Corrientes, Argentina. El área estudiada pertenece al dominio fitogeográfico amazónico, Provincia Paranen$\mathrm{se}^{2}$. El sitio de muestreo era un curso de agua temporal con vegetación acuática, rodeado de pastura natural frecuentada por bovinos.

Los caracoles colectados fueron acondicionados convenientemente y transportados vivos en bolsas de polietileno gruesas con agua del lugar cerradas herméticamente. En el laboratorio, algunos caracoles fueron relajados, muertos y conservados en Railliet-Henry ${ }^{17}$. Los caracoles fueron identificados en base a características morfológicas de la conchilla y órganos internos ${ }^{16}$.

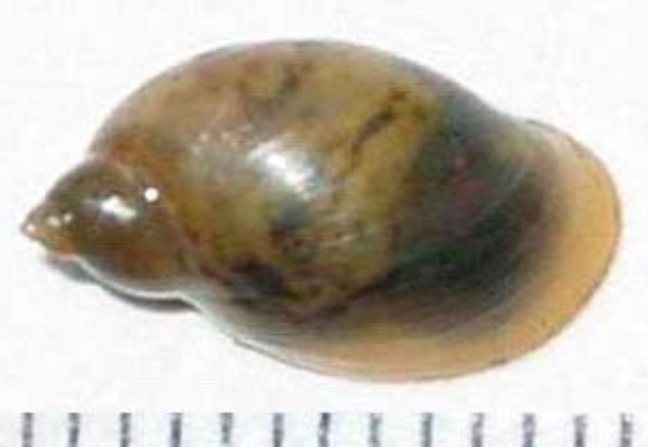

Figura 1. Pseudosuccinea columella. La escala indica milímetros.

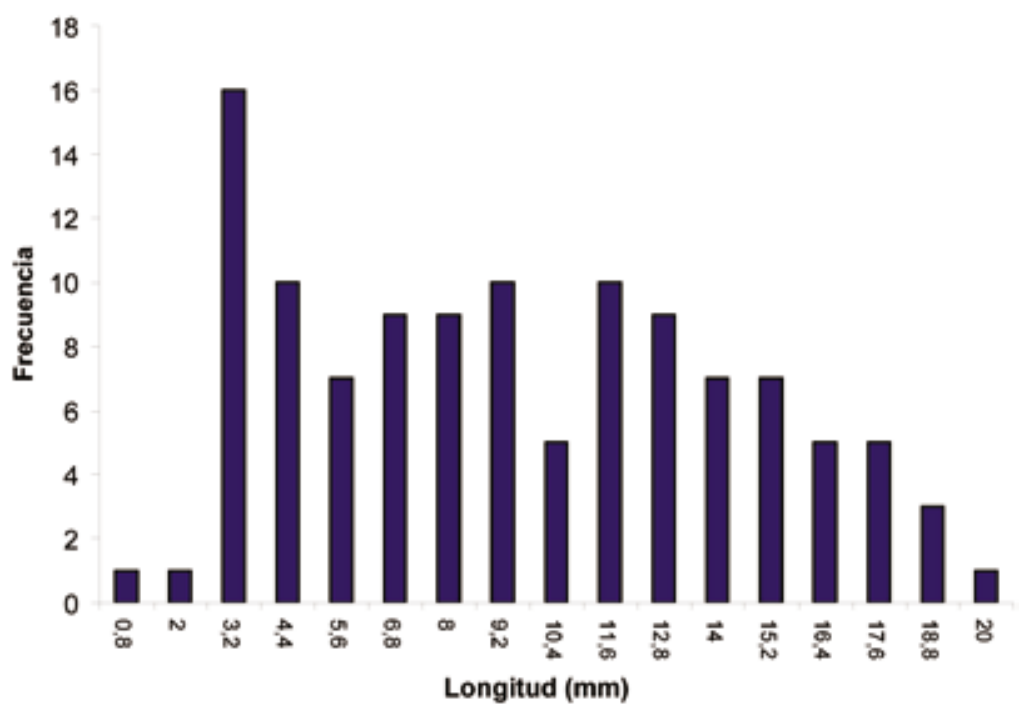

Figura 2. Número de ejemplares de Pseudosuccinea columella distribuidos en distintos intervalos según longitud de caparazón.
La longitud de la conchilla de los caracoles fue medida desde el ápice hasta el margen anterior. Otros ejemplares, similares a aquellos identificados taxonomicamente, fueron disecados para verificar la presencia de cercarias de $F$. hepatica en vísceras. La determinación de $F$. hepatica fue basada en la morfología de las cercarias obtenidas de los caracoles y confirmada a partir de adultos recuperados de ratas Wistar infestadas experimentalmente con metacercarias.

\section{RESULTADOS}

Los 115 caracoles colectados fueron identificados como Pseudosuccinea columella. La longitud de la conchilla varió entre 0,8 y $21,3 \mathrm{~mm}$, con una media de $10,0 \mathrm{~mm}$. La mayoría de los caracoles estaban maduros sexualmente (> 6,5 mm: $84 \%$ ). El intervalo de tamaño más frecuente fue el de $3,2 \mathrm{~mm}$ (Figura 2).

Se examinaron parasitologicamente 108 caracoles de los cuales $8(7,4 \%)$ estaban naturalmente infestados exclusivamente con cercarias de $F$. hepatica. Los adultos recuperados en las ratas Wistar infestadas experimentalmente fueron identificados como $F$. hepatica.

\section{DISCUSIÓN}

Cuando se comenzó a estudiar la fasciolosis en la Provincia de Corrientes en la década de 1970, la enfermedad estaba restringida al área centro-oeste, donde Lymnaea viatrix y Lymnaea palustris eran los únicos hospedadores intermediarios responsables de la transmisión del parásito ${ }^{10}$. Desde entonces, nuevos casos de fasciolosis se detectaron en mataderos del norte y de otras partes de la Provincia, como lo indican los registros de decomisos en distintas plantas de faena ${ }^{12,13}$.

En el norte y nordeste de la Provincia de Corrientes, la presencia de $L$. viatrix nunca fue documentada, mientras que $P$. columella se halló primero en la localidad de Ituzaingó ${ }^{15}$, luego en Berón de Astrada ${ }^{18}$ y en el presente trabajo en Santo Tomé. La amplia distribución de $P$. columella y la alta prevalencia de infestación encontrada en este trabajo, así como en Berón de Astrada, indicarían el establecimiento de un ciclo de transmisión enzoótica en esta zona.

Uno de los factores más importantes que posiblemente contribuyó a este escenario fue el aumento del nivel de agua en los esteros del Iberá, después de haberse puesto en funcionamiento la represa de Yacyretá, a partir de 1989. Al formase el lago de la represa, el agua se escurrió a las partes bajas del terreno, afectando especialmente al Departamento de Ituzaingó ${ }^{~}$. Estas crecientes pudieron haber provocado un aumento 
en la cantidad de habitats adecuados para el desarrollo de $P$. columella.

La prevalencia de $P$. columella naturalmente infestada por $F$. hepatica registrada en Santo Tomé resultó alta $(7,4 \%)$ al igual que en Berón de Astrada $(8,8 \%)^{18}$ en el año 2003. A su vez, estas cifras son mayores a las reportadas en ciertos estados de Brasil como Minas Gerais $(5,2 \text { y } 3,9 \%)^{4}$; Río de Janeiro $(2,4 \%)^{19}$; Sao Paulo $(5,26 \text { y } 1,22 \%)^{14,25}$ y Río Grande do Sul $\left(3,3 \%{ }^{23}\right.$. Este último dato es particularmente interesante debido a que la Provincia de Corrientes limita con el estado de Río Grande do Sul.

Según los resultados obtenidos hasta la actualidad, todo parece indicar que $P$. columella podría jugar un papel epizootiológico importante en la transmisión de $F$. hepatica en la Provincia de Corrientes y el nordeste de Argentina.

\section{REFERENCIAS}

1. Blanco D, Parera A. 2001. La inundación silenciosa. El aumento de las aguas en los esteros del Iberá: la nueva amenaza de la represa Yacyretá, Ed. Fundación Vida Silvestre Argentina, Buenos Aires, $27 \mathrm{p}$.

2. Cabrera AL, Willink A. 1980. Biogeografía de América latina. En: Monografía 13, Serie Biología (OEA Ed.), Washington, p. 69-75.

3. Castellanos ZA, Landoni NA. 1981. La familia Lymnaeidae Rafinesque, 1815, en la Argentina. En: Fauna de agua dulce de la República Argentina (Ringuelet RA, Ed.), PROFADU, CONICET, Buenos Aires, p. 55-82.

4. Coelho LH, Lima WS. 2003. Population dynamics of Lymnaea columella and its natural infection by Fasciola hepatica in the State of Minas Gerais, Brazil. J Helminthol 77: 7-10.

5. Del Huerto LM, Hernández S, Acuña AM, Nari A. 1996. Fascioliasis en la República Oriental del Uruguay. Anales Congreso Latinoamericano de Parasitología, Santiago, Chile.

6. Fujii TU, Oliveira SM. 1996. Ocorrência de caramujos Lymnaea columella naturalmente infestados por Fasciola hepatica, no município de Miracatu, Vale do Ribeira, SP. Jornada Paulista de Parasitologia, Sao Paulo, p. 17.

7. Heinzen T, Castro O, Pepe C, Ibarburu A. 1994. Lymnaea columella como hospedador intermediario de Fasciola hepatica en Uruguay. Anales XXII Jornadas Uruguayas de Buiatria, Paysandú, Uruguay.

8. Hubendick B. 1951. Recent Lymnaeidae: their variation, morphology, taxonomy, nomenclature and distribution. Kungl Svensk Vetenskaps Handl 3: 1-223.

9. Larrea HC, Flórez MF, Vivar RG, Huamán PM, Velásquez JP. 2007. Hospederos intermediarios de Fasciola hepatica en Perú. Revista Horizonte Médico 7: 39-46.

10. Lombardero OJ, Moriena RA, Racioppi O, Quiroz L. 1979. Binomia de Limnaea spp. en la Provincia de Corrientes. Rev Vet 2: 5-19.

11. Malek EA, Chrosciechowski P. 1964. Lymnaea (Pseudosuccinea) columella from Venezuela, and notes on the distribution of Pseudosuccinea. Nautilus 78: 54-56.
12. Moriena RA, Racioppi O, Alvarez JD, Lombardero OJ. 1999. Fasciola hepatica y otros trematodos de los rumiantes. Prevalencia y distribución en la Provincia de Corrientes. Reunión de Comunicaciones Científicas y Tecnológicas UNNE (Corrientes, Argentina). http://www.unne.edu. ar/cyt/cyt2000.htm.

13. Moriena RA, Racioppi O, Alvarez JD, Lombardero OJ. 2001. Fasciola hepatica y otros trematodos en bovinos: Prevalencia según decomisos en plantas faenadoras en la Provincia de Corrientes (Argentina). Vet Arg 18: 493-498.

14. Oliveira SM, Fujii TU, Sposito Filha E, Martins AM. 2002. Ocorrência de Lymnaea columella Say, 1817 infectada naturalmente por Fasciola hepatica (Linnaeus, 1758), no Vale do Ribeira, São Paulo, Brasil. Arq Inst Biol 69: 29-37.

15. Paraense WL. 1982. Lymnaea viatrix and Lymnaea columella in the neotropical region: a distributional outline. Mem Inst Oswaldo Cruz 77: 181-188.

16. Paraense WL. 1983. Lymnaea columella in northern Brazil. Mem Inst Oswaldo Cruz 78: 477-482.

17. Paraense WL. 1984. Lymnaea diaphana: a study of topotypic specimens. Mem Inst Oswaldo Cruz 79: 75-81.

18. Prepelitchi L, Kleiman F, Pietrokosvky S, Moriena RA, Racioppi O, Alvarez JD, Winsnivesky MC. 2003. First report of Lymnaea columella Say, 1817 (Pulmonata: Lymnaeidae) naturally infected with Fasciola hepatica (Linaeus, 1758) (Trematoda: Digenea) in Argentina. Mem Inst Oswaldo Cruz 98: 889-891.

19. Rezende HE, Araújo JL, Gomes PA, Nurenberg S, Neto MP, Oliveira GP, Mello RP. 1973. Notas sobre duas espécies de Lymnaea Lamark, 1799, hospedeiros intermediários de Fasciola hepatica L. no Estado do Rio de Janeiro (Mollusca, Gastropoda, Basommatophora, Lymmaeidae). Arq Univ Fed Rural 3: 21-23.

20. Salazar L, Estrada VE, Velásquez LE. 2006. Effect of the exposure to Fasciola hepatica (Trematoda: Digenea) on life history traits of Lymnaea cousini and Lymnaea columella (Gastropoda: Lymnaeidae). Exp Parasit 114: 77-83.

21. Schafranski NL, Vianna SS, Saber AF. 1977. Levantamento de focus de Fasciola hepatica Linnaeus, 1758, no Vale do Paraíba, Estado de São Paulo; encontro de Lymnaea columella Say, 1817, naturalmente infectada. Conferencia Anual da Sociedade Paulista de Medicina Veterinaria, São Paulo, p. 103.

22. Scott MI. 1953. Sobre Limnaeidae argentinos. Physis 20: 401-408.

23. Silva Santos IC, Laranja RJ, Costa NC, Bothelo GA, Ceresér VH, Martins JR. 1987. Lymnaea columella Say, 1817 interagindo na disseminação de fasciolose na Estação Exp. Zootécnica de Dom Pedrito, RS. A Hora Veterinaria 6: 42-48.

24. Souza CP, Magalhães KG, Jannotti Passos LK, Pereira GC, Ribeiro F, Katz N. 2002. Aspects of the maintenance of the life cycle of Fasciola hepatica in Lymnaea columella in Minas Gerais, Brazil. Mem Inst Oswaldo Cruz 97: 407-410.

25. Ueta MT. 1980. Ocorrência de infecção natural de Fasciola hepatica Linnaeus, 1758 em Lymnaea columella Say, 1817 no vale do Paraíba, SP, Brasil. Rev Saude Publica 14: 230-233. 